\title{
Consolidated Management of Business Process Variants
}

\author{
Marlon Dumas \\ University of Tartu, Estonia \\ marlon.dumas@ut.ee
}

In business processes within large organizations, one will often find variations stemming from segmentation along customer types, product lines, business units or geographical regions. For example, a business process for handling claims in an insurance company will vary depending on whether the claim relates to a car accident, a property damage or a personal incident. Also, in an insurance company that operates in several jurisdictions or countries, one is likely to observe variations in the way insurance claims are handled across these political boundaries. Similarly, in company mergers, the merged organization often ends up with multiple models describing "equivalent" processes previously executed separately in each organization prior to their merger.

One way of managing such variations is to treat each process variant as a separate process, and to model and manage each variant separately and independently of one another. Under this approach, the above insurance company would model and manage its car accident claims handling process separately from its property damage claims handling process, without any connection between them. This approach has the risk of leading to redundancies, inconsistencies and inefficiencies due to fragmentation. The other extreme approach is to manage an entire family of process variants together, as if they were variations within a single consolidated process. This approach however has the risk of leading to higher complexity, as the consolidated ("uber") process is hard to understand, implement and evolve. Moreover, organizational pressures may sooner or later cause the consolidated process to diverge into multiple ones if there is no strict framework for managing its evolution.

It remains an open question how to best manage the tradeoff between maintaining families of process variants separately versus maintaining them in a consolidated manner, and how to effectively capture and maintain consolidated business process models.

A range of approaches exist that strike different tradeoffs and can be placed at various points in the spectrum from fragmented to consolidated management of process variants. In this talk, we will review some existing techniques. In particular, the talk will discuss three consolidated process management approaches: consolidation based on "shared subprocesses", consolidation based on "configurable models", and consolidation based on "model synchronization". 